effects $^{12}$. Further research on ILC2 subsets may lead to a better understanding of their mechanism of action.

A decade after their discovery, ILCs are still at the heart of intense research, with their various roles in cancer development and response to treatment being slowly uncovered. With their expression of PD-1, ILC2s are becoming a new therapeutic target for checkpoint blockade that could enhance anticancer immune responses driven by dendritic cells and $\mathrm{CD}^{+} \mathrm{T}$ cells but also by eosinophils, as demonstrated by Jacquelot et al. Boosting ILC2 functions using IL-33 in association with anti-PD-1 may represent a promising therapeutic combination in melanoma. Unleashing the anticancer capacities of ILC2s could have positive repercussions for the treatment of patients with melanoma.

\section{Melanie Bruchard ${ }^{1}$ and \\ Francois Ghiringhelli (D) 1,2,3凶 \\ ${ }^{1}$ UMR INSERM, 1231 Dijon, France. ${ }^{2}$ Centre Georges \\ Francois Leclerc, Dijon, France. ${ }^{3}$ University of \\ Burgundy-Franche Comté, Dijon, France. \\ $凶_{e-m a i l: f g h i r i n g h e l l i @ c g f l . f r}$}

Published online: 7 June 2021

https://doi.org/10.1038/s41590-021-00945-x
References

1. Jacquelot, N. et al. Nat. Immunol. https://doi.org/10.1038/s41590021-00943-z (2012).

2. Monticelli, L. A. et al. Nat. Immunol. 12, 1045-1054 (2011).

3. Bie, Q. et al. J. Immunol. Res. 2014, 923135 (2014).

4. Trabanelli, S., Chevalier, M. F., Derré, L. \& Jandus, C. Semin. Immunol. 41, 101276 (2019).

5. Trabanelli, S. et al. Nat. Commun. 8, 593 (2017).

6. Chevalier, M. F. et al. J. Clin. Invest. 127, 2916-2929 (2017).

7. Schuijs, M. J. et al. Nat. Immunol. 21, 998-1009 (2020).

8. Long, A. et al. J. Immunol. 201, 3456-3464 (2018)

9. Ikutani, M. et al. J. Immunol. 188, 703-713 (2012).

10. Kim, J. et al. J. Immunol. 196, 2410-2423 (2016).

11. Moral, J. A. et al. Nature 579, 130-135 (2020).

12. Wang, S. et al. Cell Res. 30, 610-622 (2020).

\title{
SARS-CoV-2-specific hotspots in virus-host interaction networks
}

\author{
A multimodal proteomic analysis of the perturbations that SARS-CoV-2 and SARS-CoV induce in infected human \\ lung epithelial cells reveals common and distinct immune-evasive and pathobiological mechanisms used by these \\ coronaviruses.
}

\section{Herbert B. Schiller, Merlijn van Breugel and Martijn C. Nawijn}

B asic research on coronaviruses, spurred by the first SARS coronavirus (SARS-CoV) epidemic in 2002-2003, has set the stage for the unprecedented effort to understand SARS-CoV-2 biology, which is needed to face the COVID-19 pandemic. Viruses evade innate immune responses by hijacking basic cellular pathways and modulating key host factors and signaling pathways $^{1}$. In Nature, Stukalov et al..$^{2}$ use state-of-the-art mass spectrometry to study the multidimensional effects that SARS-CoV and SARS-CoV-2 have on the proteome and transcriptome of infected human lung epithelial cells. The unique strength of this study is the use of a single experimental platform, the A549 lung epithelial carcinoma cell line, for the generation of time-resolved transcriptomic, proteomic, phosphoproteomic and ubiquitinomic data in response to both SARS-CoV and SARS-CoV-2 infection. Moreover, this cell line is also used to chart the perturbation of the cellular proteome mediated by each of the individual viral gene products. To this end, the authors identify pathways (from the Reactome database) that connect the interactome of each viral gene product with the proteomic changes observed in response to the overexpression of that gene product (the 'effectome') using network diffusion analysis (Fig. 1). Integration of these different data modalities yields an unprecedented level of detail about the functional interactions of SARS-CoV-2 and SARS-CoV with the host proteome upon infection of human lung epithelial cells.

Some of the main results of this study stem from the direct comparison of SARS-CoV-2 to its predecessor SARS-CoV. Despite their homology, the gene products from these two related coronaviruses substantially differ in their interactions with host factors. For example, the SARS-CoV-2 ORF3 and ORF8 proteins displayed a range of interactions with host factors that were not observed with the homologous gene products of SARS-CoV ${ }^{2}$. Interestingly, the SARS-CoV-2-specific interactome can be used to infer mechanistic insight into the higher transmission rates and specific hallmark symptoms of COVID-19. For instance, SARS-CoV-2 ORF8 uniquely interacts with intermediates of the TGF- $\beta$ and extracellular matrix signaling pathways, which are known factors in fibrosis development, one of the long-term sequelae of COVID-19 (ref. ${ }^{3}$ ). Indeed, in lungs from patients who succumbed to the disease, TGF- $\beta$ signaling has been mapped to $C T H R C 1^{+}$'pathological' fibroblasts, which were suggested to drive fibrosis in COVID-19 (ref. ${ }^{4}$ ).

To systematically explore the protein networks responding to the expression of individual viral gene products, the authors use a network diffusion approach (Hierarchical HotNet ${ }^{5}$, which so far has been mainly used in cancer research. This analysis identifies the subnetworks in the host cell proteome affected by the individual viral proteins. Projecting these subnetworks onto the multiomic, time-resolved data obtained after viral infection allows for a detailed description of the transcriptomic, proteomic and post-translational changes induced by SARS-CoV-2 in these biological pathways (Fig. 1). For instance, specific functions for SARS-CoV-2 ORF3 were observed in the regulation of autophagy. Expression of SARS-CoV-2 ORF3 not only induced the accumulation of autophagy receptors but also led to the increased ubiquitination of 
a

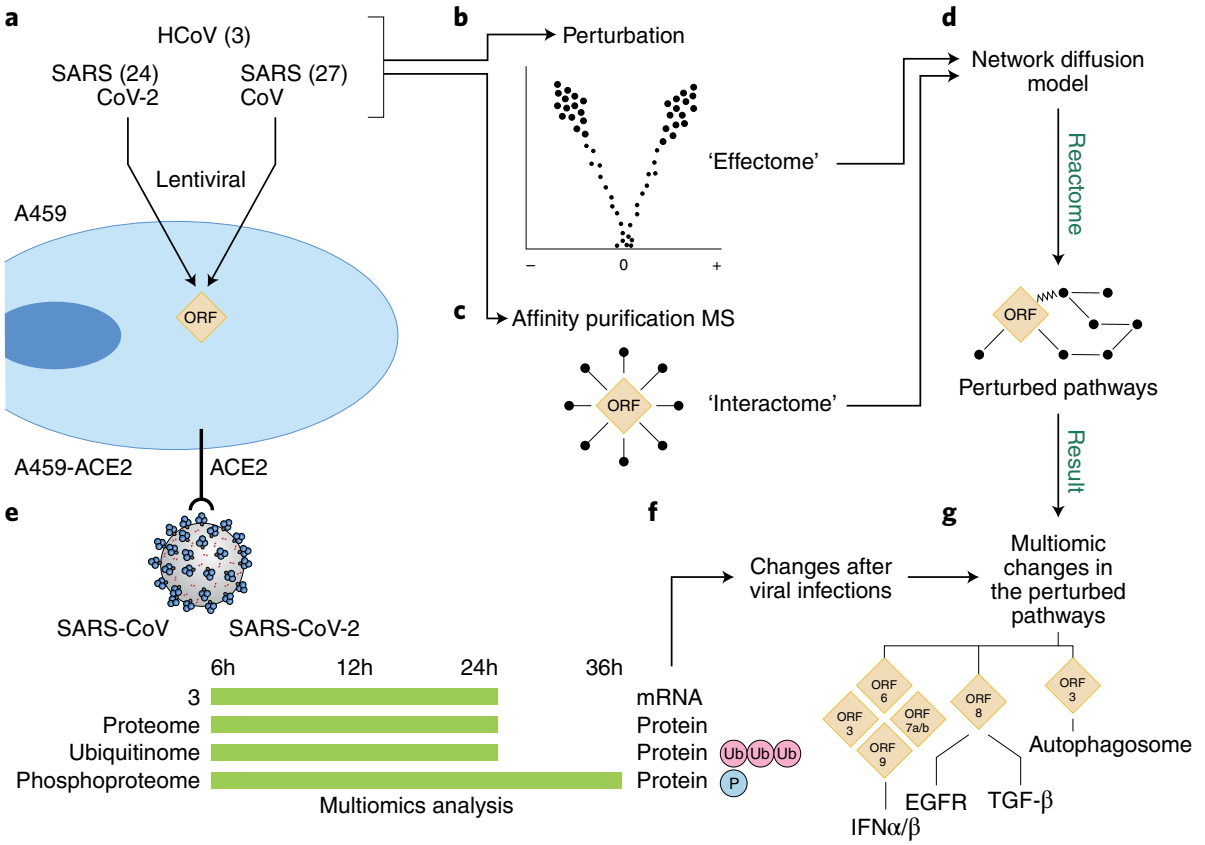

Fig. 1 | Multiomic analysis of coronavirus-induced cellular changes. Stukalov et al. generate two discrete datasets. a, Viral proteins from SARS-CoV, SARS-CoV-2 and HCoV are individually introduced by lentiviral transfection into A549 cells. b. Mass spectrometry (MS) analysis is used to chart changes in the cell proteome in response to the perturbation caused by each viral protein (the 'effectome'). c, Affinity purification MS is used to identify the host factors interacting with each viral protein. d, These results are combined by network diffusion modeling (using the Reactome database as a reference) to identify the pathways that respond to each viral protein. $\mathbf{e}$, The authors also perform a time-resolved transcriptomic, proteomic, ubiquitinomic and phosphoproteomic analysis of the response to infection with SARS-CoV and SARS-CoV-2 in A549 cells. f,g, This analysis generates a multiomic reference dataset that is used to characterize the transcriptomic and proteomic changes and the post-translational modifications ( $\mathbf{f})$ in the pathways that respond to the individual viral proteins $(\mathbf{g})$. This integrative analysis offers an unprecedented level of detail on the cellular response to coronavirus infection and identifies a SARS-CoV-2-specific modulation of the TGF- $\beta$ pathway by ORF8, as well as the regulation of the autophagosome by ORF3. Moreover, regulation of the innate immune response by ORF3, ORF6, ORF7a/b and ORF9a is charted in great detail.

several autophagy-associated factors. The affinity purification mass spectrometry data for SARS-CoV-2 ORF3 shows a direct interaction with the HOPS complex, which mediates autophagosome-lysosome fusion, suggesting that SARS-CoV-2 may actively suppress autophagosome-mediated protein degradation upon infection. The expression of one known target of the autophagosome, APOB, was increased in A549 cells after SARS-CoV-2 infection or ORF3 overexpression, which was suggested to be a possible mechanism to explain the increased risk of arterial thrombosis observed in COVID-19 (refs. ${ }^{2,6}$ ). As such, the data generated in this study provide a valuable resource for hypothesis-driven interrogation of proteomic changes after SARS-CoV-2 infection that can also be further integrated with (single-cell) transcriptomic or proteomic datasets from patient studies that explore the pathogenesis of COVID-19.
The study by Stukalov et al. also provides further insight into the modulation of the innate immune response by SARS-CoV-2. While infection of respiratory epithelial cells with SARS coronaviruses induces relatively modest type I and type III interferon (IFN) responses in comparison to other respiratory viruses, it drives a marked NF- $\kappa \mathrm{B}$-dependent proinflammatory chemokine and cytokine response ${ }^{7,8}$. In side-by-side comparisons using matched lung tissue explants, SARS-CoV infection induces significantly higher type I and III IFN responses as compared to SARS-CoV-2 (ref. ${ }^{9}$ ). Moreover, the magnitude of the airway epithelial type I IFN response after SARS-CoV-2 infection inversely correlates with the severity of COVID-19 (ref. ${ }^{10}$ ). The data from Stukalov et al. confirm the previously reported suppression of the type I IFN response by both SARS-CoV and SARS-CoV-2 (ref. ${ }^{7,8}$ ), mapping this effect to ORF3, ORF6, ORF7a/b and ORF9a. The SARS-CoV-2-specific ORF8-dependent modulation of the TGF- $\beta$ pathway may also be relevant in this respect, as TGF- $\beta$ suppresses the induction of type I IFN responses and facilitates viral replication in airway epithelial cells ${ }^{11}$. Stukalov et al. also report that a proinflammatory signature is prominently induced at every omics level analyzed. Interestingly, SARS-CoV had a more marked induction of proinflammatory cytokines and chemokines than SARS-CoV-2 in matched lung tissue explants ${ }^{9}$, which may offer insights into the more severe course of disease in SARS-CoV. The multiomics data presented in this resource may yet reveal many additional insights into the mechanisms of innate immune evasion by SARS-CoV-2, which probably contribute to both the severity of disease and the ease of transmission, two key aspects of the current pandemic.

Stukalov et al. also present a comprehensive overview of changes in two relevant post-translational modifications (ubiquitination and phosphorylation) after SARS-CoV and SARS-CoV-2 infection. More than half of the marked alterations in the host cell ubiquitinome and phosphoproteome after infection were shared between the two viruses, with most viral proteins also ubiquitinated and phosphorylated after infection. The viral protein interactome data reveal that a number of viral gene products interact with host E3 ligases, deubiquitinating enzymes and kinases, suggesting that viruses evolve in the context of post-translational modifications of their proteins by the host ${ }^{12}$. Of particular interest may be the extensive multiubiquitination of the $S$ protein, which was conserved for both viruses. It will be important to follow up on the potential regulatory functions of these new post-translational modifications of the SARS S protein.

Finally, the authors explored the relevance of their data and model system to reveal the potential antiviral activity of drugs known to selectively target the cellular pathways perturbed by SARS-CoV-2 infection. In this screen, several specific inhibitors that are currently in use for unrelated disorders were found to increase viral growth, whereas several other candidates showed antiviral activity. Among the latter were the selective matrix metalloprotease (MMP)2 and MMP9 inhibitors prinomastat and marimastat, which suppressed SARS-CoV-2 but not SARS-CoV replication. These observations currently lack a mechanistic basis but are of interest for further study, especially given the aforementioned SARS-CoV- 
2 -specific modulation of the TGF- $\beta$ pathway as well as the increased expression of extracellular matrix proteins, such as fibrinogen (FG)A, FGB and fibronectin, and remodeling factors such as SERPINE1, after SARS-CoV-2 infection.

This study has generated a comprehensive overview of the multiomic changes that occur in response to SARS-CoV-2 infection in the A549 human lung carcinoma cell line. The use of this cell line as the single platform for all analyses has the risk of missing cell-type-specific protein-protein interactions relevant to primary lung epithelial cell types or other lineages, such as myeloid or endothelial cells. Also, the lentiviral overexpression system used to characterize the interactome and effectome of the viral proteins could lead to the mislocalization or dysregulation of viral protein expression, rendering false-positive interactions or exaggerated perturbation results. Thus, future validation using complementary methods such as in situ proximity ligation assays in infected human lung tissue or primary cell culture models will be important. However, by cross-referencing protein networks (rather than individual proteins) identified in the network diffusion analysis with the transcriptomic, proteomic and post-translational changes observed after viral infection, the authors focus on the most relevant changes in cellular pathways provoked by SARS-CoV-2 infection. A systems biology approach such as that employed in this study can generate relatively unbiased insights. Integration of multilayered datasets, such as the one presented here, beyond comparisons of results obtained in analyses of a single omics layer remains a challenge that requires more advanced statistical and computational techniques, which will allow integrative analysis across the various levels of omics data and will advance a more holistic understanding of disease.

Herbert B. Schiller (D)', Merlijn van Breugel 2,3,4 and Martijn C. Nawijn (iD 4,5凶

${ }^{1}$ Comprehensive Pneumology Center, Institute of Lung Biology and Disease, Helmholtz Zentrum München, Member of the German Center for Lung Research (DZL), Munich, Germany. ${ }^{2}$ MIcompany, Amsterdam, the Netherlands. ${ }^{3}$ Department of Pediatric Pulmonology and Pediatric Allergology, Beatrix Children's Hospital, University of Groningen, University Medical Center Groningen, Groningen, the Netherlands. ${ }^{4}$ GRIAC Research Institute, University Medical Center Groningen, Groningen, the Netherlands. ${ }^{5}$ Department of Pathology and Medical Biology, University of Groningen, University Medical Center Groningen, Groningen, the Netherlands.

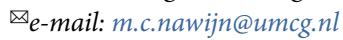

Published online: 21 June 2021

https://doi.org/10.1038/s41590-021-00963-9

References

1. Bösl, K. et al. Front. Immunology 10, 2186 (2019).

2. Stukalov, A. et al. https://doi.org/10.1038/s41586-021-03493-4 (2021).

3. George, P. M., Wells, A. U. \& Jenkins, R. G. Lancet Respir. Med. 8 , 807-815 (2020)

4. Melms, J. C. et al. Nature https://doi.org/10.1038/s41586-02103569-1 (2021)

5. Reyna, M. A., Leiserson, M. D. M. \& Raphael, B. J. Hierarchical HotNet: identifying hierarchies of altered subnetworks. Bioinformatics 34, i972-i980 (2018).

6. Bonaventura, A. et al. Nat. Rev. Immunol. 21, 319-329 (2021).

7. Blanco-Melo, D. et al. Cell 181, 1036-1045.e9 (2020).

8. Hadjadj, J. et al.369, 718-724 (2020)

9. Chu, H. et al. Clin. Infect. Dis. 71, 1400-1409 (2020)

10. Ziegler, C. G. K. et al. Preprint at bioRxiv https://doi. org/10.1101/2021.02.20.431155 (2021).

11. Denney, L., Branchett, W., Gregory, L. G., Oliver, R. A. \& Lloyd, C. M. Mucosal Immunol. 11, 523-535 (2018).

12. Fung, T. S. \& Liu, D. X. Future Virol. 13, 405-430 (2018).

Competing interests

The authors declare no competing interests. 